5-1996

\title{
Storage and Delivery Constrained Unit Commitment
}

\author{
Y. Al-kalaani \\ Cleveland State University \\ F. Eugenio Villaseca \\ Cleveland State University, f.villaseca@csuohio.edu \\ F Renovich Jr
}

Follow this and additional works at: https://engagedscholarship.csuohio.edu/enece_facpub

Part of the Power and Energy Commons

How does access to this work benefit you? Let us know!

\section{Publisher's Statement}

(C) 1996 IEEE. Personal use of this material is permitted. Permission from IEEE must be obtained for all other uses, in any current or future media, including reprinting/republishing this material for advertising or promotional purposes, creating new collective works, for resale or redistribution to servers or lists, or reuse of any copyrighted component of this work in other works.

\section{Original Citation}

Al-kalaani, Y., Villaseca, F. E., \& Renovich, J. F. (1996). Storage and Delivery Constrained Unit Commitment. IEEE Transactions on Power Systems : a Publication of the Power Engineering Society, 11, 2, 1059-1066.

\section{Repository Citation}

Al-kalaani, Y.; Villaseca, F. Eugenio; and Renovich, F Jr, "Storage and Delivery Constrained Unit Commitment" (1996). Electrical Engineering \& Computer Science Faculty Publications. 88.

https://engagedscholarship.csuohio.edu/enece_facpub/88

This Article is brought to you for free and open access by the Electrical Engineering \& Computer Science Department at EngagedScholarship@CSU. It has been accepted for inclusion in Electrical Engineering \& Computer Science Faculty Publications by an authorized administrator of EngagedScholarship@CSU. For more information, please contact library.es@csuohio.edu. 


\title{
STORAGE AND DELIVERY CONSTRAINED UNIT COMMITMENT
}

\author{
Y. Al-kalaani, Student Member IEEE
}

\author{
F. E. Villaseca, Member IEEE \\ Department of Electrical Engineering \\ Cleveland State University
}

\begin{abstract}
A general formulation and the development of a dynamic programming algorithm to solve a fuel-constrained unit commitment problem is presented. The system under consideration has oil units with storage constraints, gas units with delivery constraints, and unconstrained coal units. An optimal approach to transfer the fuel delivery constraints into unit capacity limits using a closed-form dual dispatch is introduced. As a result, the gas units can be viewed as fuel-unconstrained, thus greatly reducing complexity. It is shown that the optimization problem, which requires that storage levels be parameterized, leads to two different dispatch rules. The oil units are dispatched to maximize the power they generate for a binding fuel amount or cost, whereas the gas and coal units are economically dispatched to provide for the remaining power. In addition to the standard constraints, the system is required to meet a minimum system spinning reserve. Test results are provided to illustrate the merits of the proposed method.
\end{abstract}

Keywords: Short-term unit commitment, dynamic programming, fuel constraints, spinning reserve, economic dispatch, dual dispatch, fuel-limited capacity units.

\section{INTRODUCTION}

In power systems, fuel availability is constrained by various factors such as contractual obligations and storage restrictions. For instance, take-or-pay contracts, which due to economics and limitations of the fuel transmission systems, have usually added provisions that specify, at any given period, the minimum amount of fuel that must be delivered with an upper cap on fuel deliverability. Also, regulatory or physical restrictions impose limitations on the amount of fuel that can be stored. These fuel considerations, in addition to the traditional constraints, such as system demand, unit generation capacities, and spinning reserve, can extensively complicate the unit commitment problem and its embedded economic dispatch. Several methods have been proposed to solve the fuel-constrained unit commitment problem which can be classified into two trade-off categories. In the first [1-6] fuel constraints are integrated into a single problem which can be solved for a global solution. In the other category [7-11], the scheduling problem is separated into two, namely fuel dispatch and unit commitment which are iteratively solved using suitable optimization techniques. Although both approaches may accommodate for different fuel constraints, the first is applicable to greatly simplified problems, while the other can only yield suboptimal solutions. Converting fuel and/or emission constraints into the corresponding generator limits as mentioned in $[12,13]$ is only possible on an individual basis, that is, if the constraints on each unit are known. However, this method will fail when the constraints, such as those imposed by the take-or-pay contracts, are shared among all the affected units.

In this paper, a new approach to solve the unit commitment problem using a dynamic programming based algorithm is presented. The novelty of this method is that system fuel constraints can be optimally transformed into new bounds on the lower and upper power output of the generating units using the dual dispatch concept which renders the problem fuelunconstrained. The system under consideration has oil units fed from a common tank with a constant hourly flow rate input, gas units fed from a common pipeline subject to hourly minimum and maximum fuel flow, as well as daily minimum (take-or-pay) and maximum fuel consumption, and fuel-unconstrained coal units. This study shows that the use of dynamic programming, which requires that storage level be parameterized, induces two different dispatch problems. At any given stage, the oil units are dispatched to maximize the power they generate for a fixed cost, whereas the coal and the gas units are dispatched to generate the power not provided by the oil units, to minimize their production cost. In addition to the standard constraints, the system is required to meet a minimum system spinning reserve, which must be considered by both dispatch problems.

\section{PROBLEM FORMULATION}

Unit commitment is a constrained optimization problem whose solution determines the set of generating units, among those owned by the utility, that should be connected to the grid at any hourly interval over a period of time, lasting from a day 
to a week, such that an objective function -usually, cost of operation-, is minimized. To state the problem mathematically we need the following definitions.

\section{A. Definitions}

Let the time horizon be divided into $M$ hourly stages. Then the set of all stages is $K=\{0, I, 2, \ldots ., k, \ldots . ., M]$.

Assume that a utility has $N$ generating units. Then the set of all units, or the universal set, is $I=(1,2, \ldots, i, \ldots . ., N]$.

Since a generating unit may be on or off let the status of unit $i$, or commitment variable, be defined by the binary set $d_{i}$ $\epsilon(0,1)$. The set of all units may be divided into two mutually exclusive sets:

$I_{O N}=\left\{i \in I \mid d_{i}=1\right\}$, the set of units on-line, and

$I_{o f}=\left\{i \in I \mid d_{i}=0\right)$, the set of units off-line that is, satisfying $I_{O N} \cup I_{O F}=I$ and $I_{O N} \cap I_{O F}=\Phi$.

Since the status of any unit is described by a digit in the binary set, then the set of all possible unit combinations at each stage is given by $U=(1,2, \ldots, j, . ., J)$

where: $J=2^{N}-1$.

Given the status $d_{i}$ of every unit $i \in I$, the unit combination number may readily be obtained by the binary to decimal transformation $j=\sum_{i \in I} 2^{i-1} d_{i}=\sum_{i \in I_{O N}} 2^{i-1}$.

The dynamic programming formulation requires that storage be parameterized into $L$ levels. Let the set of all parameterized levels be $W=(1,2, \ldots, l, \ldots, L)$. Therefore the state of the system with fuel storage constraints is a function of three parameters, unit combination $j$, level $\boldsymbol{l}$, and stage $\boldsymbol{k}$. Let the state variable be defined by $x\left(j_{k}, l_{k}\right)$, then the set of all possible states over the scheduling horizon is $S=\{1,2, . ., x, . ., X\}$, where: $\boldsymbol{X}=\boldsymbol{M} \times \boldsymbol{N} \times \boldsymbol{J} \times \boldsymbol{L}$.

If $p_{i}$ is the power allocated to unit $i$ then the fuel cost for this unit, assuming quadratic functions, is

$$
C_{i}\left(p_{i}\right)=a_{i} p_{i}^{2}+b_{i} p_{i}+c_{i} \quad(\$ / h)
$$

where: $a_{i}, b_{i}$, and $c_{i}$ are the cost coefficients of unit $i$.

Since the amount of fuel consumed depends on the difference between the level at the current stage $l_{k}$ and the level at the previous stage $l_{k \cdot l}$, then the production cost associated with state $x\left(j_{k}, l_{k}\right)$ at any given $j, l$, and $k$ is

$$
\boldsymbol{P C O S T}\left[x\left(j_{k}, l_{k}\right), l_{k-1}\right]=\sum_{i=1}^{N} C_{i}\left(p_{i}\right) d_{i}
$$

The transitional cost associated with the change from state $x\left(j_{k-1}, l_{k-1}\right)$ to state $x\left(j_{k}, l_{k}\right)$ is

$$
\begin{gathered}
\operatorname{TCOST}\left[x\left(j_{k-1}, l_{k-1}\right), x\left(j_{k}, l_{k}\right)\right]= \\
\sum_{i=1}^{N}\left|d_{i}\left(l_{k}\right)-d_{i}\left(l_{k-1}\right)\right| T C_{i}\left[l_{k}, t_{i}\left(l_{k-1}\right)\right]
\end{gathered}
$$

where:

$\left.T C_{i l}.\right]$ is the transitional cost (start-up or shutdown) $(\$ / h)$ of unit $\boldsymbol{i}$ at stage $\boldsymbol{k}$ and level $\boldsymbol{l}_{\boldsymbol{k}}$ from stage $\boldsymbol{k}-\boldsymbol{l}$ and level $\boldsymbol{l}_{\boldsymbol{k}-\boldsymbol{l}}$, $t_{i}($.$) is the number of hours that unit i$ at stage $k-I$ and level $l_{k-1}$ has been on or off up to stage $k-1$.

\section{B. Problem Statement}

The commitment problem can be stated as

$$
\begin{aligned}
\arg \min Z(x) & =\sum_{k=1}^{H} \operatorname{PCOST}\left[x\left(j_{k}, l_{k}\right), l_{k-1}\right] \\
& +\operatorname{TCOST}\left[x\left(j_{k-1}, l_{k-1}\right), x\left(j_{k}, l_{k}\right)\right]
\end{aligned}
$$

where: $\boldsymbol{Z}($.$) is the total cost of operation (\$)$.

This optimization problem is subject to the following constraints:

\section{Power Balance Constraint

$$
P G\left[x\left(j_{k}, l_{k}\right), l_{k-1}\right]=P D_{k}(M W), \forall x
$$

where:

$$
P G[.]=\sum_{i=1}^{N} p_{i} d_{t}
$$

is the total power allocated to state $x\left(j_{k}, l_{k}\right)$ given $l_{k-1}$, and $\mathbf{P D}_{\mathbf{k}}$ is the system demand plus transmission losses at stage $k$. These losses are included as a percent of the system demand.

\section{Unit Capacity Constraints}

$$
P_{i, \text { MIN }} \leq p_{i} \leq P_{i, \max }(M W), \forall i, \forall l, \forall k
$$

$$
\begin{aligned}
& \text { 3. Spinning Reserve Constraint } \\
& \qquad \operatorname{SSR}\left[x\left(j_{k}, l_{k}\right), l_{k-1}\right] \geq \operatorname{MSSR}(\mathrm{MW}), \forall x
\end{aligned}
$$

where:

$$
S S R[.]=\sum_{i \in I_{\text {ON }}} \min \left(M S R_{i}, P_{i, \text { MAX }}-p_{i}\right)
$$

$M S R_{i}$ is the maximum spinning reserve for unit $i$, $M S S R$ is the minimum system spinning reserve.

\section{Fuel Storage Constraints}

$$
q_{s}\left(l_{k}, l_{k-1}\right)=q_{C}+\frac{Q\left(l_{k-1}\right)-Q\left(l_{k}\right)}{T} \geq 0
$$

where:

$$
L_{\text {MNN }} \leq l \leq L_{\text {MUX }} \quad\left(m^{3}\right), \quad \forall l
$$

$q_{s}($.$) is the amount of fuel \left(m^{3} / h\right)$ that can be drawn out of the tank given levels $l_{k}$ and $l_{k \cdot 1}$.

$Q\left(l_{k}\right)$ is the amount of fuel $\left(m^{3}\right)$ stored at level $l_{k}$ and stage $k$, $Q\left(l_{k-1}\right)$ is the amount of fuel $\left(m^{3}\right)$ stored at level $l_{k-l}$ and stage $k-1$,

$q_{c}$ is a constant fuel flow rate $\left(m^{3} / h\right)$ into the tank,

$T$ is the time interval $(h)$ between two consecutive stages,

$L_{M A X}$ is the tank maximum level, and

$L_{M I N}$ is the tank minimum level not necessarily zero. 
Typically, there are two types of delivery constraints associated with fuel contracts which must be considered:

\section{Hourly Fuel Delivery Constraints}

$$
q_{\min } \leq q_{d}(k) \leq q_{\max } \quad\left(m^{3} / h\right), \forall k
$$

where:

$\boldsymbol{q}_{d}(\boldsymbol{k})$ is the amount of fuel to be allocated at each stage $\boldsymbol{k}$, and the constraints imposed by the fuel transmission system $q_{\text {min }}$ is the hourly minimum fuel rate that must be consumed, $q_{\max }$ is the hourly maximum fuel deliverabilty rate,

\section{Daily Fuel Delivery Constraints}

$$
Q_{M I N} \leq \sum_{k=1}^{M} q_{d}(k) \leq Q_{M A X} \quad\left(m^{3}\right)
$$

where:

$Q_{M N}$ is the daily minimum fuel (take-or-pay), and

$Q_{\text {MAX }}$ is the daily maximum fuel amount that can be delivered.

In addition to the above constraints, the following must also be considered:

7. Unit Minimum-Up and Downtime Constraints

8. Must Run Unit Constraints

9. Units Assigned to Fixed Generation

10. Unit Startup and Shutdown Constraints

11. Crew Constraints

11. Changes in Unit Status and Characteristics.

The recursion required, at every stage and level, to solve this optimization problem using dynamic programming is given by

$$
\begin{aligned}
\operatorname{CCOsT}\left[x\left(j_{k}, l_{k}\right)\right]= & \min \left\{\operatorname{CCOsT}\left[x\left(j_{k-1}, l_{k-1}\right)\right]\right. \\
& +\operatorname{PCOST}\left[x\left(j_{k}, l_{k}\right), l_{k-1}\right] \\
& \left.+\operatorname{TCOsT}\left[x\left(j_{k-1}, l_{k-1}\right), x\left(j_{k}, l_{k}\right)\right]\right\}
\end{aligned}
$$

where:

$\operatorname{CCOST}[$.$] is the cumulative cost (\$)$ associated with every state $x\left(j_{k}, l_{k}\right)$ subject to all the problem constraints.

\section{FUEL-CONSTRAINED ECONOMIC DISPATCH}

When considering fuel storage constraints, the standard economic dispatch which uses Kuhn-Tucker optimality conditions to minimize system production cost, must be modified to include these equality constraints. Assume a system with $N$ generating units, $N_{s}$ of which are supplied from a common storage tank, $N_{D}$ from a common delivery pipeline, and $N_{v}$ are fuel unconstrained units. That is $N=N_{s}+N_{b}+$ $N_{U}$. Let the set of all units supplied from the storage tank be $I_{s}=\left\{1,2, \ldots, i, \ldots, N_{s}\right\}$, the set supplied from delivery pipeline be $I_{D}=\left\{N_{S}+I, \ldots, i, . ., N_{S}+N_{D}\right\}$, and the set of fuel-unconstrained units be $I_{U}=\left\langle N_{\mathrm{S}}+N_{D}+1, \ldots, i, \ldots, N\right)$. Therefore the set of all units is $I=I_{S} \cup I_{D} \cup I_{U^{*}}$ If a set of on-line units $I_{O N}$ in state $x\left(j_{k}, l_{k}\right)$ is to be dispatched at stage $k$ to meet a system load $P D_{k}$, then the production cost for this state is

$$
\begin{gathered}
P \operatorname{COST}\left[x\left(j_{k}, l_{k}\right), l_{k-1}\right]=\sum_{i=1}^{N_{s}} C_{i}\left(p_{i}\right) d_{i} \\
+\sum_{i=N_{s}+1}^{N_{s}+N_{D}} C_{i}\left(p_{i}\right) d_{i}+\sum_{i=N-N_{U}}^{N} C_{i}\left(p_{i}\right) d_{i}
\end{gathered}
$$

Consider that $\boldsymbol{I}_{s}$ units, on-line at stage $\boldsymbol{k}$, draw fuel from the storage at a total consumption rate $(5)$ which is fixed by the level $l_{k}$ being considered and by each level $l_{k-1}$, the total cost of consuming fuel is also fixed at

$$
\sum_{i=1}^{N_{t}} C_{i}\left(p_{j}\right) d_{i}=F C\left(l_{k}, l_{k-1}\right)
$$

where:

$$
F C\left(l_{k}, l_{k-1}\right)=C S \cdot q_{s}\left(l_{k}, l_{k-1}\right)
$$

in which $C S$ is the fuel cost per cubic meter $\left(\$ / m^{3}\right)$.

The economic dispatch problem is to determine the value of $p_{i}, i \in I=I_{s} \cup\left\{I_{D} \cup I_{v}\right\}$, such that (8) is minimized subject to the system constraints (disregarding for the moment fuel delivery and system spinning reserve constraints which will be considered later):

$$
P D_{k}-\sum_{i=1}^{N_{s}} p_{i} d_{i}-\sum_{i=N_{i}+1}^{N} p_{i} d_{i}=0
$$

and

$$
\sum_{i=1}^{N_{L}} C_{l}\left(p_{l}\right) d_{i}-F C\left(l_{k}, l_{k-1}\right)=0
$$

Since (10) and (11) are equality constraints, they can be incorporated in the cost function via LaGrange multipliers $\lambda$ and $\gamma$ to form the objective function

$$
\begin{aligned}
& J=F C\left(l_{k}, l_{k-1}\right)+\sum_{i=N_{s}+1}^{N} C_{l}\left(p_{i}\right) d_{i} \\
& +\lambda\left(P D_{k}-\sum_{i=1}^{N_{s}} p_{i} d_{i}-\sum_{i=N_{s}+1}^{N} p_{i} d_{i}\right) \\
& +\frac{\lambda}{\gamma}\left[\sum_{i=1}^{N_{s}} C_{i}\left(p_{i}\right) d_{i}-F C\left(l_{k}, l_{k-1}\right)\right]
\end{aligned}
$$

from which the optimality conditions are obtained as

$$
\gamma=\frac{d C_{i}\left(p_{i}\right)}{d p_{i}} d_{i}(\$ / M W h), \quad i \in I_{s}
$$

and

$$
\lambda=\frac{d C_{i}\left(p_{i}\right)}{d p_{i}} d_{i}(\$ / M W h), i \in I_{D} \cup I_{U}
$$

Notice that (12) and (13) are independent of each other, that is $I_{S} \cap\left\{I_{D} \cup I_{U}\right\}=\Phi$. 


\section{OPTIMAL DISPATCH POLICY}

Since the fuel cost (9) is already fixed by the storage levels being considered at each stage, the optimal dispatch policy, which has the overall objective of meeting the system demand at minimum production cost, would be to generate as much power as possible out of this fixed cost so that $I_{D}$ and $I_{V}$ units have less power to generate for the remaining load not provided by the $I_{s}$ units. Of course, the above policy is optimal only if such power maximization dispatch exists.

The equal incremental cost functions can be used to achieve different dispatch objectives. For instance a dispatch using (13) and (10) is the well known economic dispatch which minimizes production cost for a given load, whereas a dispatch using (12) and (11) is a dual dispatch which maximizes power generation for a given cost, closed-form solutions for the dispatch problem may be derived as follows.

From (13), the power allocated to unit $i$ is determined by taking the derivative of (1) with respect to $p_{i}$ as

$$
p_{i}=\frac{\lambda-b_{i}}{2 a_{i}} \quad i \in I_{O N}
$$

By substituting (14) into (2), the system load may be written as

$$
P D_{k}=\sum_{i \in I_{\text {ON }}} \frac{\lambda-b_{i}}{2 a_{i}}
$$

from which a closed-form expression for $\lambda$ is obtained

$$
\lambda=\frac{1}{\sum_{i \in I_{\text {ow }}} \frac{1}{a_{i}}}\left(2 P D_{k}+\sum_{i \in I_{\text {ow }}} \frac{b_{i}}{a_{i}}\right)
$$

In a similar fashion, by substituting (14) into (1) and (9) a closed-form expression for $\gamma$ can be readily derived as

$$
\gamma=\sqrt{\frac{F C(.)+\sum_{i \in I_{\text {oN }}}\left(\frac{b_{i}^{2}}{4 a_{i}}-c_{i}\right)}{\sum_{i \in I_{\text {ON }}} \frac{1}{4 a_{i}}}}
$$

Thus knowledge of $\lambda$ and $\gamma$ uniquely specifies the generation level of each unit, since the system load, fuel cost, and the cost coefficients in (15) and (16) are known.

Since (12) and (13) are decoupled as indicated earlier, the dispatch of the on-line units can be performed separately as follows:

- Dispatch $I_{s}$ units using (16). This results in the maximization of the total power $P G_{s}$ generated by these units for a fixed cost given by (11)

$$
P G_{s}=\sum_{i=1}^{N_{s}} p_{i} d_{i}=\sum_{i=1}^{N_{s}} \frac{\gamma-b_{i}}{2 a_{i}} d_{i}
$$

that is, any power allocation other than (17) will result in less total generation for the same fuel cost, and

- Dispatch the $I_{D}$ and $I_{U^{\prime}}$ units using (15). This is a standard economic dispatch, where these units assume the generation not provided by $I_{S}$ units, that is

$$
\sum_{i=N_{s}+1}^{N} p_{i} d_{i}=P D_{k}-P G_{s}
$$

These dispatch problems are also constrained by the limits on unit generation (3), so that if one or several units in either set reach their limit values, the optimal strategy requires that the remaining units in the set operate so as to satisfy their respective optimality conditions [14].

\section{Changing Dispatch objectives}

Consideration of the fuel delivery limits requires that constraints (6) be checked every time the on-line units in set $I_{D}$, which must not be empty, are dispatched. However, since (7) is a daily constraint, a cumulative fuel consumption associated with each state must also be checked at each stage. If the right side of $(7)$ is violated at any stage, then the corresponding state is infeasible and therefore must be removed from the dynamic optimization path. On the other hand. if (6) is violated and $I_{U^{\prime}}=\Phi$, then the dispatch of these units is not feasible. Furthermore, if (6) is violated but $\boldsymbol{I}_{V} \neq \Phi$ in that unit combination, then the dispatch of the $I_{D}$ units may be rendered feasible as follows:

- If (6) is violated at its lower limit, then the cost of fuel to be consumed by $I_{D}$ units is set equal to this limit, that is

$$
\sum_{i=N_{s}+1}^{N_{D}} C_{i}\left(p_{i}\right) d_{i}=C D \cdot q_{\mathrm{min}}
$$

where: $C D$ is the fuel cost per cubic meter $\left(\$ / m^{3}\right)$.

- If (6) is violated at its upper limit, then the cost of fuel to be consumed by $I_{D}$ units is set equal to this limit, that is

$$
\sum_{i=N_{s}+1}^{N_{D}} C_{i}\left(p_{i}\right) d_{i}=C D \cdot q_{\max }
$$

Since (19) and (20) are fixed costs, the dispatch objectives of $I_{D}$ units must be changed from cost minimization, as posed earlier, to power maximization.

Therefore, the dispatch of the $I_{D}$ and $I_{U}$ units will be modified as follows:

- Dispatch $I_{D}$ units using (16) with the objective of maximizing their total generation $P G_{D}$ for a fixed cost given by (19) or (20) depending on the case

$$
P G_{D}=\sum_{i=N_{s}+1}^{N_{D}} p_{i} d_{i}=\sum_{i=N_{s}+1}^{N_{D}} \frac{\gamma-b_{i}}{2 a_{i}} d_{i}
$$

- Dispatch $I_{v}$ units using (15) to economically meet the remaining load not provided by $\boldsymbol{I}_{S}$ and $\boldsymbol{I}_{D}$ units, that is

$$
\sum_{i=N-N_{U}}^{N} p_{i} d_{i}=P D_{k}-P G_{s}-P D_{D}
$$




\section{FUEL-LIMITED CAPACITY UNITS}

Although the above dispatching scheme can perfectly account for any type of fuel constraints encountered in this study, its practical implementation is somewhat cumbersome because the dispatch objectives have to be constantly changing. every time constraints (6) are not met. Since violations of these constraints cannot be known a priori, it is required that the online units be dispatched first before any dispatch policy can be adopted. With thousands of dispatches to consider at every stage and level over the scheduling horizon, this method is highly inefficient in the use of computer time and memory.

An optimal but simple technique to solve this complex multi-dispatching problem is presented here by using the closed-form dual dispatch which maximizes generation for fixed fuel cost, to incorporate the fuel delivery constraints directly into the units generation capacities, thus rendering the problem fuel-unconstrained. That is

- For fixed cost (19), the unit generation levels, as determined by (16), are to become the new minimum generation capacities for these units

$$
P_{i, M I N}^{N}=p_{i} d_{i} \quad i \in I_{D}
$$

- For a fixed cost (20), the unit generation levels, as determined by (16), are to become the new maximum generation capacities for these units

$$
P_{i, \text { MAX }}^{N}=p_{i} d_{i} \quad i \in I_{D}
$$

The computational effort, due to forms (23) and (24), is greatly reduced. Dispatches (17) and (18) could both be performed as indicated with the fuel constraints guaranteed to be met. The iterative process, which was required in steps (1922) to check the fuel delivery limits after each dispatch, is no longer needed here since $I_{V}$ units and $I_{D}$ units with their new capacity limits are now economically dispatched as fuelunconstrained, that is $I_{D} \in I_{U}$, with (6) automatically met.

\section{SPINNING RESERVE REDISPATCH}

In this study, the minimum system spinning reserve $M S S R$ is considered a hard constraint which must always be met. There are instances in which the economic dispatch of a set of units may violate constraint (4) by an amount $\Delta=M S S R$ $S S R\left[u_{k}\right]$. However, with the division of set $I_{O N}$ into two subsets defined as $I_{1}=\mid i \in I_{O N}: S R_{i}=M S R_{i} /$ and $I_{2}=\mid i \in I_{O N}: S R_{i}=$ $P_{i, M A X}-p_{i} /$, it is possible to meet MSSR by bringing up and down the generations of these sets by $U P G=\Sigma\left(/ P_{i, \text { MAX }}\right.$. $\left.\left.M S R_{i}\right\}-p_{i}\right] i \in I_{1}$ and $D N G=\Sigma\left[p_{i}-\left\langle P_{i, M A X}-M S R_{i} /\right] i \in I_{2}\right.$. respectively, if the conditions of the following theorem are satisfied.

Theorem 1 - When $\Delta>0$, the redispatch of wits $I_{1}$ and $I_{2}$ will yield a new system spinning reserve $\mathbf{S S} \boldsymbol{R}_{N} \geq \mathbf{M S S R}$ if and only if $U P G \geq \triangle$ and $D N G \geq \triangle$.

Proof: Provided in reference [15].

The conditions of Theorem 1 can only be tested on-line, that is after a dispatch has been performed. Such an a posteriori test is very inefficient in terms of computer memory and time. The following theorem provides an a priori test for feasibility.

Theorem 2 - If a set $I_{O N}$ is infeasible by violating MSSR, it can still be rendered feasible by redispatch if and only if

$$
\sum_{i \in I_{\text {ON }}} P_{i, \text { MIN }} \leq P D_{k} \leq \sum_{i \in I_{\text {ON }}} P_{i, \text { MAX }}-M S S R
$$

and

$$
\sum_{i \in I_{\text {oN }}} M S R_{i} \geq M S S R
$$

Proof: Provided in reference [16].

Due to the inclusion of storage constraints, conditions (25) and (26) become only necessary to meet (4). However, these conditions can be tested off-line. that is before the start of the unit commitment algorithm, to eliminate all the infeasible sets that cannot meet constraints $(2,3,4)$, thereby truncating drastically the number of decisions to be considered in the solution space. In other words, the necessary and sufficient conditions of Theorem I need only tested on those sets $I_{O N}$ that satisfy (25) and (26).

Specifically, the proposed spinning reserve redispatch algorithm, which exploits the separability of the fuel constrained model, proceeds as follows.

Once a dispatch of a set of on-line units, satisfying (25) and (26), is performed as described above, then constraint (4) is checked for compliance. If $M S S R$ is not met, then the redispatch of these units will depend on the number and type of units involved as follows. If the set $I_{O N} \in\left\{I_{S} \cup I_{U}\right\}$ includes:

- One $I_{S}$ unit and one $I_{V}$ unit, redispatch is not feasible, that is the conditions of Theorem $I$ are not satisfied, and this $I_{O N}$ is discarded.

- One $I_{s}$ unit and more than one $\boldsymbol{I}_{U}$ unit, dispatch the $\boldsymbol{I}_{U}$ units if feasible.

- One $I_{v}$ unit and more than one $I_{S}$ unit, dispatch the $I_{S}$ units if feasible.

- More than one unit in both sets, dispatch $I_{s}$ units if feasible. This may result in two different situations:

1) The $I_{s}$ units are capable of meeting (4) by themselves, and the $I_{V}$ units are dispatched to meet the remaining generation, or

2) The $I_{S}$ units while increasing their contribution to the spinning reserve, it is insufficient to meet $M S S R$, and the $I_{v}$ units are redispatched to provide for the remaining generation and spinning reserve. 


\section{SYSTEM DATA}

Although the computer program can accommodate any number of units, for illustration purposes, the system chosen in this study, has ten units. 3 oil units, 3 gas units, and 4 coal units. The unit characteristics are given in Table 1.

The parameters of the production, startup and shutdown costs are given in Table II. The oil units are fed from a common storage tank which receives oil at a constant rate of $1000(\$ / h)$. For illustration, storage is parameterized into five levels specified in terms of cost as 2000, 2500, 3000, 3500, and $4000(\$)$. The initial and final tank levels over a $24(h)$ time horizon are required to be $3000(\$)$. The gas units are fed from a common pipeline which continuously delivers gas at a minimum rate of $500(\$ / h)$ but not to exceed $2500(\$ / h)$. The daily minimum and maximum gas costs are set to $12,000(\$)$ and $24,000(\$)$ respectively. The system load profile over a 24 (h) horizon is shown in Table III. The system spinning reserve $M S S R$ is set to $750(M W)$ which is equal to the capacity of the largest unit in the system.

TABLE I. Unit characteristics

\begin{tabular}{|rcrrrrrrrr|}
\hline \multicolumn{2}{|c|}{ No TYPE } & PMAX PMIN & MSR & MU & MD & \multicolumn{2}{c|}{ PUS HON HOF } \\
\hline 1 & 011 & 400 & 80 & 100 & 2 & 1 & 0 & 0 & 2 \\
2 & 011 & 400 & 85 & 100 & 2 & 1 & 0 & 0 & 2 \\
3 & 011 & 450 & 90 & 120 & 3 & 2 & 0 & 0 & 2 \\
4 & Gaa & 420 & 100 & 100 & 0 & 0 & 0 & 0 & 2 \\
5 & Gas & 280 & 75 & 120 & 0 & 0 & 0 & 0 & 2 \\
6 & Gas & 320 & 80 & 90 & 0 & 0 & 1 & 4 & 0 \\
7 & Coal & 443 & 125 & 250 & 2 & 1 & 1 & 3 & 0 \\
8 & Coal & 750 & 250 & 430 & 3 & 2 & 1 & 4 & 0 \\
9 & Coal & 543 & 125 & 120 & 2 & 1 & 0 & 0 & 2 \\
10 & Coal & 443 & 125 & 60 & 2 & 1 & 0 & 0 & 1 \\
\hline
\end{tabular}

Notes:

NO: number, TYPE: fuel type; PMAX: maximum generation capacity $(M W)$; PMIN: minimum generation capacity $(M W)$; MSR: maximum spinning reserve $(M W)$; MU: minimum up time ( $k$ ); MD: minimum down time $(h)$; PUS: 0,1 , indicating unit previously off or on respectively; HON: number of hours unit has been on previously; HOF: number of hours unit has been off previously.

TABLE II. Parameters, Startup, and Shutdown Costs

\begin{tabular}{|cccccccc|}
\hline NO & $a$ & $b$ & $c$ & $c C S$ & cR & EC & SD \\
\hline 1 & .00500 & 1.000 & 50 & 100 & .2 & 15 & 30 \\
2 & .00250 & 2.000 & 30 & 100 & .1 & 20 & 40 \\
3 & .00350 & 1.500 & 40 & 120 & .2 & 8 & 50 \\
4 & .00489 & 2.345 & 69 & 120 & .1 & 22 & 80 \\
5 & .00443 & 2.335 & 72 & 115 & .1 & 17 & 65 \\
6 & .00480 & 2.328 & 92 & 110 & .2 & 18 & 60 \\
7 & .00148 & 1.114 & 82 & 130 & .1 & 21 & 85 \\
8 & .00100 & 1.000 & 50 & 110 & .2 & 19 & 72 \\
9 & .00135 & 1.100 & 49 & 100 & .1 & 22 & 70 \\
10 & .00138 & 1.114 & 72 & 100 & .2 & 19 & 75 \\
\hline
\end{tabular}

Notes:

$\overline{\mathrm{SC}(\mathrm{T})}=\mathrm{CCS}\left(1-\mathrm{e}^{-\mathrm{T} \cdot \mathrm{CR}}\right)+\mathrm{FC}(\$)$, is the startup cost function

$\mathrm{T}=$ time the unit has been down $(h)$

CCS $=$ cost of a cold start $(h)$

$\mathrm{CR}=$ cooling rate $\left(h^{\cdot t}\right)$

$F C=$ fixed cost involved with a startup $(\$)$

$\mathrm{SD}=$ fixed cost involved with a shutdown $(\$)$.
TABLE III. Optimal Hourly Allocations for Case 1

\begin{tabular}{|c|c|c|c|c|}
\hline $\begin{array}{l}\text { stage } \\
(\mathrm{h})\end{array}$ & $\begin{array}{l}\text { LOAD } \\
\text { (MW) }\end{array}$ & $\begin{array}{c}\text { Gas } \\
(\$ / h)\end{array}$ & $\frac{011}{(\$ / h)}$ & $\begin{array}{c}\text { RESBRVB } \\
\text { (MW) }\end{array}$ \\
\hline $\begin{array}{l}1 \\
2 \\
3 \\
4 \\
5 \\
6 \\
7 \\
8 \\
9 \\
10 \\
11 \\
12 \\
13 \\
14 \\
15 \\
16 \\
17 \\
18 \\
19 \\
20 \\
21 \\
22 \\
23 \\
24\end{array}$ & $\begin{array}{l}1200 \\
1300 \\
1450 \\
1550 \\
1810 \\
1950 \\
2200 \\
2310 \\
2450 \\
2600 \\
2850 \\
2900 \\
3000 \\
3030 \\
3020 \\
2850 \\
2350 \\
2100 \\
2050 \\
2000 \\
1900 \\
1810 \\
1720 \\
1215\end{array}$ & $\begin{array}{r}500.0 \\
500.0 \\
500.0 \\
500.0 \\
500.0 \\
500.0 \\
500.0 \\
500.0 \\
500.0 \\
624.4 \\
1083.5 \\
993.4 \\
991.4 \\
1087.5 \\
1055.0 \\
1083.5 \\
500.0 \\
500.0 \\
500.0 \\
500.0 \\
500.0 \\
500.0 \\
500.0 \\
500.0\end{array}$ & $\begin{array}{r}500 \\
500 \\
1000 \\
1000 \\
1000 \\
1000 \\
1000 \\
1000 \\
1000 \\
1000 \\
1000 \\
1500 \\
1500 \\
1500 \\
1500 \\
1000 \\
1000 \\
1000 \\
1000 \\
1000 \\
1000 \\
1000 \\
500 \\
500\end{array}$ & $\begin{array}{r}800.0 \\
900.0 \\
1000.0 \\
1000.0 \\
1000.0 \\
1000.0 \\
950.0 \\
873.5 \\
757.0 \\
789.8 \\
750.0 \\
831.1 \\
750.0 \\
750.0 \\
750.0 \\
750.0 \\
843.0 \\
990.5 \\
1000.0 \\
1000.0 \\
1000.0 \\
1000.0 \\
900.0 \\
900.0\end{array}$ \\
\hline
\end{tabular}

\section{COMPUTER RESULTS AND DISCUSSION.}

The computer code is a full dynamic programming implementation of the algorithm. A VAX series 6000 , Model 410 , computer was utilized to solve the fuel-constrained unit commitment problem. To clearly illustrate the implication of these different fuel constraints, only three cases were presented in this paper. Case 1 has system constraints as provided in section VIII. The results are presented in Table III where for each stage, system load, fuel cost rates, and reserve are provided. For Case 2, the daily minimum and maximum fuel delivery costs were increased to $16,000(\$)$ and $32,000(\$)$ respectively, whereas in Case 3 , the hourly minimum gas rate is increased from $500(\$ / h)$ to $800(\$ / h)$ with the daily limits as those of Case 1. For the entire horizon, the oil costs, the gas costs, and the optimal costs which include those of the coal units are presented in Table IV for comparison. Finally optimal schedule for Case 1 is presented in Table V.

As shown in Table MI, the hourly fuel costs of the oil units match exactly the fuel cost rates which are fixed by the tank levels between stages, whereas, the hourly fuel costs of the gas units are within the allowable cost limits. Since in this study, gas is relatively more expensive than coal, the optimal solution calls for the gas units to generate at the minimum take-or-pay cost, unless the system demands more generation to meet the load at peak hours. As expected, the system spinning reserve is shown to be higher than the minimum requirement when redispatch is not needed, and exactly equal to $750(M W)$ when redispatch is performed.

Since the tank initial and final levels are identical, the oil cost for all three cases, shown in Table IV to be $24,000(\$)$, is equal to the total cost of fuel fed to the tank during $24(h)$. For 
TABLE IV. Total Operation Costs

\begin{tabular}{|cccc|}
\hline $\begin{array}{c}\text { Cage } \\
\text { No. }\end{array}$ & $\begin{array}{c}\text { Oil cost } \\
(\$)\end{array}$ & $\begin{array}{c}\text { Gas Coat } \\
(\$)\end{array}$ & $\begin{array}{c}\text { Optimal Cost } \\
(\$)\end{array}$ \\
\hline 1 & 24,000 & 15,359 & 105,444 \\
\hline 2 & 24,000 & 16,226 & 106,109 \\
\hline 3 & 24,000 & 20,634 & 107,685 \\
\hline
\end{tabular}

TABLE V. Optimal Schedule for Case 1

\begin{tabular}{|c|cccccccccc|}
\cline { 2 - 9 } \multicolumn{1}{c|}{} & \multicolumn{8}{c|}{ Ualt } & Number \\
\cline { 2 - 8 } Stage & 1 & 2 & 3 & 4 & 5 & 6 & 7 & 8 & 9 & 10 \\
\hline 1 & 1 & 0 & 1 & 0 & 1 & 0 & 1 & 1 & 1 & 0 \\
2 & 1 & 0 & 1 & 0 & 1 & 0 & 1 & 1 & 1 & 1 \\
3 & 1 & 1 & 1 & 0 & 1 & 0 & 1 & 1 & 1 & 1 \\
4 & 1 & 1 & 1 & 0 & 1 & 0 & 1 & 1 & 1 & 1 \\
5 & 1 & 1 & 1 & 0 & 1 & 0 & 1 & 1 & 1 & 1 \\
6 & 1 & 1 & 1 & 0 & 1 & 0 & 1 & 1 & 1 & 1 \\
7 & 1 & 1 & 1 & 0 & 1 & 0 & 1 & 1 & 1 & 1 \\
8 & 1 & 1 & 1 & 0 & 1 & 0 & 1 & 1 & 1 & 1 \\
9 & 1 & 1 & 1 & 0 & 1 & 0 & 1 & 1 & 1 & 1 \\
10 & 1 & 1 & 1 & 1 & 1 & 0 & 1 & 1 & 1 & 1 \\
11 & 1 & 1 & 1 & 1 & 1 & 1 & 1 & 1 & 1 & 1 \\
12 & 1 & 1 & 1 & 1 & 1 & 1 & 1 & 1 & 1 & 1 \\
13 & 1 & 1 & 1 & 1 & 1 & 1 & 1 & 1 & 1 & 1 \\
14 & 1 & 1 & 1 & 1 & 1 & 1 & 1 & 1 & 1 & 1 \\
15 & 1 & 1 & 1 & 1 & 1 & 1 & 1 & 1 & 1 & 1 \\
16 & 1 & 1 & 1 & 1 & 1 & 1 & 1 & 1 & 1 & 1 \\
17 & 1 & 1 & 1 & 0 & 1 & 0 & 1 & 1 & 1 & 1 \\
18 & 1 & 1 & 1 & 0 & 1 & 0 & 1 & 1 & 1 & 1 \\
19 & 1 & 1 & 1 & 0 & 1 & 0 & 1 & 1 & 1 & 1 \\
20 & 1 & 1 & 1 & 0 & 1 & 0 & 1 & 1 & 1 & 1 \\
21 & 1 & 1 & 1 & 0 & 1 & 0 & 1 & 1 & 1 & 1 \\
22 & 1 & 1 & 1 & 0 & 1 & 0 & 1 & 1 & 1 & 1 \\
23 & 1 & 0 & 1 & 0 & 1 & 0 & 1 & 1 & 1 & 1 \\
24 & 1 & 0 & 1 & 0 & 1 & 0 & 1 & 1 & 1 & 1 \\
\hline
\end{tabular}

Notes:

$1=$ unit on-line; $0=$ unit off-line.

Case 2, the gas cost is shown to be greater than in Case 1 due to the increase in the daily fuel delivery limits which results, as expected, in a higher optimal cost. Since the optimal costs shown in Table IV are different, the optimal schedules for cases 2 and 3 (which are not included due to space limitation), are also different.

\section{APPLICATIONS TO LARGE SCALE SYSTEMS}

Since fuel costs command a large portion of a utility's operating budget, slight variations from optimal solutions can result over time in considerable wasteful expenditures.

Dynamic programming (DP) algorithms guarantee optimality of solutions, but are affected by the "curse of dimensionality", that is by the number of unit combinations to be considered in the solution process. In spite of the ever increasing speed and memory of computers, at affordable cost, the efficiency of DP algorithms should always be a goal.

There are three means by which computational time and memory requirements of DP algorithms can be drastically reduced. The first is the off-line exclusion of all infeasible unit combinations, as presented here and in [16]. Another is the elimination of all iterative procedures, which we achieved in the proposed technique by problem decomposition. The third deals with techniques that limit the solution space to be searched. Although we do not address these here for reasons of space, we have presented them in [17-19], and we are in the process of implementing them for the algorithm proposed here.

The example presented above was provided for illustration purposes. The proposed algorithm may also be generalized to handle multiple fuel constraints in large scale systems. For instance, the single tank problem can easily be extended to a multiple storage problem, because the fuel to be allocated for each tank is fixed by its own parameterization and thus each problem is solvable separately. The resulting total generation is then subtracted from the load and the difference must be met by the remaining units as described above. Similarly, the single delivery point can also be extended to multiple terminals supplying several units in different plants. In this case, the fuel constraints imposed by each transmission system are transferred into the corresponding unit capacity limits using the dual dispatch theory, thus rendering these sets of units fuel-unconstrained.

\section{CONClusions}

In this paper, we have presented a new technique to solve the fuel-constrained unit commitment using dynamic programming based algorithm. The system under consideration has storage-constrained oil units, delivery-constrained gas units, and fuel-unconstrained coal units.

It was shown that the use of dynamic programming, which requires that the stored resource be parameterized, induced two different dispatch schemes. At any given stage, the oil units were dispatched to maximize the power they generate at a binding fixed fuel cost, whereas the coal and the gas units were economically dispatched to generate the remaining power, to minimize their production cost. Both dispatch strategies were modified to meet a minimum system spinning reserve requirement.

We have also shown that the complex multi-dispatching process introduced by the fuel delivery constraints was virtually eliminated by employing the dual dispatch concept which optimally converted these constraints into new unit capacity limits, thus rendering the gas unit economic dispatches fuelunconstrained.

Three examples were provided to illustrate the use of the algorithm and test results confirmed the merits of the new method.

Use of the algorithm, as described, can easily be extended to handle multiple fuel storage and delivery constraints that might be experienced in more complex systems. 


\section{ACKNOWLEDGMENTS}

The authors express their gratitude to the anonymous reviewers for their queries which have resulted in a clearer exposition.

\section{REFERENCES}

[1] Kennedy, T., S.M. Hoyt, and F. A. Buchmeier, "Intermediate Fuel Dispatch", IEEE Transactions on Power A pparatus and Systems, Vol. PAS-102, No. 7, 1983, pp. 1979-1985.

[2] Aoki, K., T. Satoh, M. Itoh, T. Ichimori, and K. Masegi, "Unit Commitment in a Large-Scale Power System induding Fuel Constrained Thermal and Pumped-Storage Hydro", IEEE Transactions on Power Systems, Vol. PWRS-2, No. 4, 1987, pp.1077-1084.

[3] Aoki, K., M. Itoh. T. Satoh, K. Nara, and M. Kanezashi, "Optimal LongTerm Unit Commitment in Large Scale Systems including Fuel Constrained Thernal and Pumped-Storage Hydro", IEEE Transactions on Power Systems, Vol. PWRS-4, No. 3, 1989, pp. 10651073.

[4] Lee, F.N., "A Fuel-Constrained Unit Commitment Method", IEEE Transactions on Power Systems. Vol. PWRS-4, No. 3, 1989, pp, 1208 1218.

[5] Lee, F.N., J. Liao, and A.M. Breipohl. "Adaptive Fuel Allocation Using Pseudo Fuel Prices", IEEE Transactions on Power Systems, Vol. PWRS7, No. 2, 1992, pp. 487-496.

[6] Cohen, A.I. and S.H. Wan, "A Method for Solving the Fuel Constrained Unit Conmitment Problem", IEEE Transactions on Power Systems, Vol. PWRS-2, No. 3, 1987,pp. 608-614

[7) Vemuri, S., A.B.R. Kumar, D.P. Hackett. J. Eisenhauer, and R. Lutgo," Fuel Resource Scheduling, Part I - Overview of an Energy Management Problem", IEEE Transactions on Power Appartus and Systems, Vol. PAS-103, No. 7, 1984. pp. 1542-1548.

[8] Kumar, A.B.R., and S. Vemuri, "Fuel Resource Scheduling, Part IIConstrained Economic Dispatch", IEEE Transactions on Power Apparatus and Systems, Vol. PAS-103. No. 7. 1984, pp. 1549-1555.

[9] Kumar, A.B.R., S. Vemuri, L.A. Gibbs, D.F. Hackett, J.T. Eisenhauer, "Fuel Resource Scheduling, Part III - The Short-Term Problem", IEEE Transactions on Power Apparatus and Systems, Vol. PAS-103, No. 7, 1984, pp. 1556-1561.

[10] Van Meeteren, H.P., "Scheduling of Generation and Allocation of Fuel, Using Dynamic and Linear Programming", IEEE Transactions on Power Apparatus and Systems, Vol. PAS-103, No. 7, 1984, pp. 15621568.

[11] Vemuri, S., and L. Lemonidis, "Fuel Constrained Unit Commitment", IEEE Transactions on Power Systems, Vol. PWRS-7, No. 1, 1992, pp 410-415.

[12] Lamont, J.W., W.G. Lesso, and K. Butz," Optimization Using a Daily Fossil Fuel Management Program", IEEE Transactions on Power Apparatus Systems, Vol. PAS-101, No. 1, 1982, pp. 87-93.

[13] Ramanathan, R.,"Emission Constrained Econonic Dispatch", 94 WM 198-2 PWRS.
[14] Wood, A.J., and B.F. Wollenberg, Power Generation, Operation, and Control, John Wiley and Sons, Inc, New York, N.Y., 1984.

[15] Renovich, F. Ir., F.E. Vilaseca, Z. Liu, and Y. Al-kalaani,"Effects of Spinning Reserve on Unit Commitment", Proceedings of the North American Power Symposium, 1993, pp. 61-68.

[16] Al-Kalaani, Y, and F.E. Villaseca, "Off-line Elimination of Unfeasible Unit Combinations in Commitment Algorithms", Proceedings of the North American Power Symposium, 1994, pp. 575-582.

[17] Fardaneh, B., and F.E. Villaseca,"Two Step Optimal Generation Scheduling", Automatica, 22, 1986, pp. 361-366.

[18] Liu, Z, F.E. Villaseca, F. Renovich, and M. Najjar,"Knowledge-Based Dynamic Progranming Algorithm for Fast Unit Commitment", Proceedings of the North American Power Symposium, 1993, pp. 135-144.

[19] Renovich Ir. F., and F.E. Villaseca,"Unit Commitment of PumpedHydro/Themal Systems", Proceedings of the North American Power Symposium, 1993, pp. 89-95. 